\title{
NON PITTING TYPE PEDAL EDEMA WITH LITHIUM: A CASE REPORT
}

Case Study

\author{
PARVATHYPRIYA C., JESLYN MARY PHILIP, CHRISTEENA GEORGE, LAKSHMI R.
}

Department of Pharmacy Practice, Amrita School of Pharmacy, Kochi, Amrita Vishwa Vidyapeetham, Amrita University, India

Email: lakshmir@aims.amrita.edu

Received: 24 Sep 2016 Revised and Accepted: 23 Nov 2016

\begin{abstract}
Objective: To report a case of lithium induced bilateral nonpitting pedal edema.
\end{abstract}

Methods: The clinical data of a bipolar affective disorder patient with current episode of mania and psychotic symptoms who experienced bilateral non pitting pedal edema with lithium.

Results: The patient was a 29 yr old female who developed bilateral non-pitting type pedal edema with lithium therapy with normal plasma lithium level $(0.72 \mathrm{mEq} / \mathrm{l})$. She is a known case of bipolar affective disorder (BPAD) was admitted to psychiatry department with episode of mania with psychotic symptoms. She had history of drug induced hypersensitivity reaction with eosinophilia and systemic symptoms (DRESS) with oxcarbazepine and so the drug was discontinued and was started on tablet lithium $400 \mathrm{mg}$ twice daily. On admission here, the dose of lithium was increased to $1200 \mathrm{mg} /$ day. The patient gradually improved but she developed bilateral non-pitting pedal edema. Serum lithium concentration was normal and there were no other early symptoms of lithium toxicity. But as the patient's distress further increased with increasing pedal edema, it was decided to stop lithium altogether and to maintain the patient on tablet quetiapine 800 mg therapy for BPAD. Within one week of stopping lithium the edema on both her feet decreased significantly. Causality was assessed by naranjo causality assessment scale and a probable relationship was obtained between lithium and pedal edema with a score of 6 .

Conclusion: This case emphasises that regular physical examination and laboratory investigations are important for patients who are on lithium therapy. Clinicians should always be careful while initiating lithium treatment in a patient with respect to the initial dose and dose escalation even after a period of successful therapy with lithium, as minor dose escalation can cause major changes in the serum lithium concentration and thereby the patient's tolerability to lithium.

Keywords: Pedal edema, Lithium, Mania

(C) 2017 The Authors. Published by Innovare Academic Sciences Pvt Ltd. This is an open access article under the CC BY license (http://creativecommons.org/licenses/by/4. 0/] DOI: http://dx.doi.org/10.22159/ijpps.2017v9i1.15353

\section{INTRODUCTION}

Lithium is widely used to treat and prevent episodes of mania in bipolar disorder. It is a mood stabiliser. It is also used for the treatment of aggression, post-traumatic stress disorder, and conduct disorder in children and as an augmenting agent for antidepressants [1]. It is available as both immediate and extended release formulations. For treatment of acute mania, it is initiated at low doses of $300 \mathrm{mg}$ three times daily or less, gradually increasing based on response and tolerability of patients. The daily dose can go up to $1800 \mathrm{mg}$ in 3 to 4 divided doses.

Lithium's mechanism of action is not well understood. It mainly affects two intracellular signalling pathways namely inositol monophosphate and glycogen synthase kinase pathway. Through the action in inositol monophosphate pathway, it reduces intracellular inositol which can be the mechanism behind mood stabilising effect of lithium. By inhibition of glycogen kinase-3, it influences energy metabolism, neuroprotection and neuroplasticity [2].

In addition to its therapeutic role, it is important to monitor patients closely, to prevent occurance of side effects as lithium has got a narrow therapeutic index. A large proportion of patients experienced at least one episode of toxicity during chronic lithium therapy [3]. Most common side effects of chronic lithium therapy is hand tremors, polydipsia, polyuria, weight gain, diarrhoea and edema in face and legs [4]. Lithium can also cause various serious side effects like cardiac arrhythmias, circulatory shock, blackout spells, headache, psychomotor retardation, acne vulgaris, and exacerbation of psoriasis, hypothyroidism, diabetes insipidus, anorexia, gastritis, oliguria, leukocytosis and tremors [7].

Cases with lithium-induced pedal edema are significant as it can even occur in normal serum lithium concentration. Here we are discussing a case of lithium-induced bilateral pedal edema in a patient who has no hepatic renal or cardiovascular disease. Mechanism of this phenomenon is not well understood as laboratory investigations were all normal. Demers et al. postulates that edema can be due to a marked increase in sodium intake which may be induced in part by mania or reduction in sodium excretory capacity induced by lithium [5].

\section{CASE REPORT}

A 29 y old female, k/c/o BPAD came with complaints of fever with rash for $2 \mathrm{~d}$ with a preceding history of starting new psychiatric medications $15 \mathrm{~d}$ prior to the episode. She was on tablet oxcarbazepine $900 \mathrm{mg}$, tablet quetiapine $600 \mathrm{mg}$, tablet zolpidem 10 $\mathrm{mg}$ and tablet clonazepam $1 \mathrm{mg}$, all for daily dosing. These medications were prescribed for a mood disorder, insomnia and to control psychotic symptoms associated with mania. On examination, she was found to have facial puffiness with rashes over the face and back. Initial lab investigations showed elevated SGOT of 41.8 IU/l (5$35 \mathrm{IU} / \mathrm{l})$, SGPT of $74.2 \mathrm{IU} / \mathrm{l}$ (5-45 IU/l), with absolute eosinophilia of $26.3 \%(1-8 \%)$. She was admitted with a provisional diagnosis of oxcarbazepine induced hypersensitivity reaction. For this she was started on symptomatic management with methylprednisolone, antihistamine and antipyretics and continued on her psychiatric medications except for oxcarbazepine which was suspected as the cause of her drug-induced hypersensitivity reaction. Dermatology consultation was sought and the same line of management was optioned. Psychiatry consultation was sought and doses of quetiapine and zolpidem were increased and also added tablet lithium $800 \mathrm{mg}$ daily for management of mania. Her facial puffiness decreased markedly and her rashes decreased and she got discharged in a stable condition with the above-mentioned management.

After one week she got admitted at the department of psychiatry due to an episode of mania with psychotic symptoms. She was continued on $20 \mathrm{mg}$ of zolpidem and $700 \mathrm{mg}$ quetiapine for insomnia and 
psychotic symptoms. Doses of methylprednisolone were gradually tapered as indicated. On this admission, the dose of lithium was increased to $1200 \mathrm{mg}$ daily for effective management of mania. Meanwhile, the patient developed bilateral non-pitting pedal edema. It was initially expected that with the tapering of methylprednisolone edema would disappear, but rather it increased.

The patient was thoroughly investigated. All laboratory parameters were within normal limits. Serum lithium estimation was done and the concentration was $0.72 \mathrm{mEq} / \mathrm{l}$ which was normal $(0.5-1.2$ $\mathrm{mEq} / \mathrm{l}$ ). Dermatology consultation was done and their advice for symptomatic relief was carried out. Medicine consultation was sought and they advised to start on tablet lasix $40 \mathrm{mg}$, but it was not given as furosemide increases serum lithium concentrations. Repeat laboratory investigations and clinical examinations were done and she was found to have oliguria and leukocytosis $(18 \mathrm{k} / \mathrm{uL})$, both being reported side effects of lithium in literature. Lower limb doppler was done to rule out deep vein thrombosis.

With time patient's distress due to edema increased and it was decided to withhold lithium as lithium-induced edema was also kept in mind in spite of its normal serum concentration. Following this edema subsided gradually and the patient received increased dose of tablet quetiapine $(800 \mathrm{mg})$ for the management of maniac symptoms. After ten days edema disappeared completely from both of her lower limbs, but she started developing increased talk and irritability. So it was decided to restart lithium at a low dose of 400 mg daily, and she was tolerating this dose well without other side effects. It was augmented with sodium valproate. Family members were educated regarding the nature of her illness. Her maniac symptoms improved well with this treatment, with no other side effects and she was discharged in a stable condition.

\section{DISCUSSION}

Lithium is a mood stabilizer which is used in the management of mania associated with bipolar disorder [7]. Chronic lithium therapy is always associated with one or more episodes of its side effects [3]. It is important to regularly monitor patients for side effects while they are on lithium as it's a drug having a narrow therapeutic index $(0.5-1.2 \mathrm{mEq} / \mathrm{l})$ [6]. Only a few reported cases of lithium-induced pedal edema is there. In this case report, side effect occurred at a normal serum lithium concentration which shows regular clinical assessment of patients is more important than laboratory investigations. Mild adverse reactions can occur even if serum lithium level is $1 \mathrm{mmol} / \mathrm{l}$. At a level of 1.5 to $2 \mathrm{mmol} / \mathrm{l}$ moderate toxicity occurs and above which severe toxicity occurs [7].

Tushar et al. described a similar case of bilateral pitting pedal edema with lithium therapy where furosemide was not given to resolve edema, rather they stopped lithium as similar to this case. Lithium's main side effect in the kidney is nephrogenic diabetes insipidus which occur as a result of an inability to concentrate urine by the nephrons due to the lithium-induced insensitivity of tubular cells towards antidiuretic hormone. Postulated treatment for this condition is with thiazide diuretics [7]. Vestergaard et al. studied clinically significant side effects of lithium in 237 patients and found that edema in face or legs induced by lithium were only seen in one tenth of the patients [4]. Demers et al. report nine consecutive cases of pretibial edema in patients who have been taking lithium carbonate where the edema resolves with spironolactone, that indicates a positive relation between tubular reabsorption of sodium and edema [5]. But in this case, edema was due to patient's intolerance to high dose lithium (1200 mg/day). So when lithium therapy was withheld, the patient improved significantly.

Later it was found that patient is tolerating low doses of lithium. This case report emphasises on the significance of narrow therapeutic index of lithium as here dose elevation $(1200 \mathrm{mg})$ is the cause of adverse drug reaction which was not seen in the lower doses $(800 \mathrm{mg})$ that the patient was taking prior to admission. Rechallenging the patient with a lower dose of lithium showed that she was tolerating it well $(400 \mathrm{mg})$. Causality was assessed by naranjo causality assessment scale and a probable relationship was obtained between lithium and pedal edema with a score of 6 .

\section{CONCLUSION}

This case emphasises that regular physical examination and laboratory investigations are important for patients who are on lithium therapy. Clinicians should always be careful while initiating lithium treatment in a patient with respect to the initial dose and dose escalation, even after a period of successful therapy with lithium, as minor dose escalation can cause major changes in the serum lithium concentration and thereby the patient's tolerability to lithium.

\section{CONFLICTS OF INTERESTS}

Declared none

\section{REFERENCES}

1. Dalvinder S, Mahindra S, Ram S. Histomorphometry of the thyroid gland in rat after lithium administration. Asian J Pharm Clin Res 2015;1:339-41.

2. Meltzer. Antipsychotic agents and lithium. In: Bertram GK, Susan BM, Anthony JT. Basic and Clinical pharmacology. 11 thed. New York: McGraw Hill; 2012. p. 487.

3. Amdisen A. Clinical features and management of lithium poisoning. Med Toxicol Adverse Drug Exper 1988;3:18.

4. Vestergaard P, Amdisen A, Schou M. Clinically significant side effects of lithium treatment: a survey of 237 patients in long term treatment. Acta Psychiatr Scand 1980;62 Suppl 3:19.

5. Demers R, Heninger G. Pretibial edema and sodium retention during lithium carbonate treatment. JAMA 1970;214:1845-48.

6. Mackichan JJ, McGory R. Interpretation of serum drug concentrations In: Mary L. Basic skills in interpreting laboratory data. $4^{\text {th }}$ ed. ASHP; 2009. p. 74.

7. Tushar S, Bir SC. Pitting type pedal edema with lithium: a case report. German J Psychiatry 2008;11 Suppl 2:76-8.

\section{How to cite this article}

- Parvathypriya C, Jeslyn Mary Philip, Christeena George, Lakshmi R. Non-pitting type pedal edema with lithium: a case report. Int J Pharm Pharm Sci 2017;9(1):323-324. 